\title{
Astrometric observations of Phobos with the SRC on Mars Express
}

\section{New data and comparison of different measurement techniques ${ }^{\star}$}

\author{
A. Pasewaldt ${ }^{1}$, J. Oberst ${ }^{1,2,3}$, K. Willner ${ }^{2}$, B. Beisembin ${ }^{2}$, H. Hoffmann ${ }^{2}$, K. D. Matz ${ }^{2}$, T. Roatsch ${ }^{2}$, G. Michael ${ }^{4}$, \\ A. Cardesín-Moinelo ${ }^{5}$, and A. E. Zubarev ${ }^{3}$ \\ 1 Department of Geodesy and Geoinformation Science, Technische Universität Berlin, Strasse des 17.Juni 135, 10623 Berlin, \\ Germany \\ e-mail: andreas.pasewaldt@tu-berlin.de \\ 2 Institute of Planetary Research, German Aerospace Center, Rutherfordstrasse 2, 12489 Berlin, Germany \\ 3 MIIGAiK Extraterrestrial Laboratory, Moscow State University for Geodesy and Cartography, Gorokhovsky pereulok 4, \\ 105064 Moscow, Russia \\ ${ }^{4}$ Institute of Geological Sciences, Freie Universität Berlin, Malteserstr. 74-100, 12249 Berlin, Germany \\ 5 European Space Astronomy Centre, European Space Agency, Villanueva de la Cañada, 28691 Madrid, Spain
}

Received 24 February 2015 / Accepted 25 April 2015

\section{ABSTRACT}

\begin{abstract}
Aims. From April 2008 to August 2011 Mars Express carried out 74 Phobos flybys at distances between 669 and $5579 \mathrm{~km}$. Images taken with the Super Resolution Channel (SRC) were used to determine the spacecraft-centered right ascension and declination of this Martian moon.

Methods. Image positions of Phobos were measured using the limb-fit and control-point measurement techniques. Camera pointing and pointing drift were controlled by means of background star observations that were compared to corresponding positions from reference catalogs. Blurred and noisy images were restored by applying an image-based point spread function in a Richardson-Lucy deconvolution.

Results. Here, we report on a set of 158 Phobos astrometric observations with estimated accuracies between 0.224 and $3.405 \mathrm{~km}$ circular w.r.t. the line of sight to the satellite. Control point measurements yield slightly more accurate results than the limb fit ones. Our observations are in good agreement with the current Phobos ephemerides by the Jet Propulsion Laboratory (JPL) and the Royal Observatory of Belgium (ROB) with mean offsets of up to $335 \mathrm{~m}$. Our data can be used for the maintenance and update of these models.
\end{abstract}

Key words. astrometry - ephemerides - planets and satellites: individual: Phobos

\section{Introduction}

The Martian moons were discovered in August 1877 by Asaph Hall, an astronomer at the United States Naval Observatory (USNO) in Washington DC. Phobos, the inner and larger of the two satellites, revolves at a mean distance of $9375 \mathrm{~km}$ from the center of Mars in a near-circular $(e=0.0151)$, nearequatorial ( $i=1.0756 \mathrm{deg}$ ) orbit with a mean period of $7.65 \mathrm{~h}$ (Jacobson 2010). Thus, it is moving far inside the synchronous orbit and is subject to tidal acceleration. Mostly due to Mars' oblateness $\left(J_{2}\right)$, its orbit is subject to apsidal and nodal precession with rates of 0.4352 and $-0.4358 \mathrm{deg} \mathrm{day}^{-1}$, respectively (Borderies \& Yoder 1990; Jacobson 2010). Phobos' rotation is tidally locked to its primary. Its constant rotational motion is superimposed by physical librations (Rambaux et al. 2012).

Since Phobos moves deep within the gravity field of Mars, its orbit is a sensitive indicator of various dynamic parameters of the Martian satellite system. Based on improved ephemerides, we may revise estimates of its mass and its C20 (dynamical polar

* Tables A.1 and A.2 are only available at the CDS via anonymous ftp to cdsarc.u-strasbg. fr $(130.79 .128 .5)$ or via

http://cdsarc.u-strasbg.fr/viz-bin/qcat?]/A+A/580/A28 flattening) and C22 (dynamical equatorial flattening) spherical harmonic coefficients (Andert et al. 2010). Furthermore, we may study the secular acceleration of Phobos that is due to tidal interactions with its primary allowing us to derive the rate of tidal dissipation within Mars and placing constraints on the mechanical properties of the planetary interior (Bills et al. 2005; Lainey et al. 2007; Jacobson 2010).

Updated orbit models of Phobos can be used to plan the acquisition of high-resolution images of the moon's surface or for the purpose of optical navigation prior to Mars orbit insertion, as demonstrated by the Optical Navigation Camera (ONC) experiment on the Mars Reconnaissance Orbiter (MRO) in February and March 2006 (Zurek \& Smrekar 2007). Finally, knowledge of the Phobos orbit is crucial for performing radio science experiments during close flybys of Phobos (Andert et al. 2010). Phobos and Deimos have been subject to numerous observation campaigns from Earth and space.

Astrometric data have been reported from Earth-based observations (e.g. catalogs compiled by Morley 1989; and Arlot \& Emelyanov 2009) ${ }^{1}$ and from space-based observations by

\footnotetext{
1 Natural Satellites Astrometric Database: http://www .imcce. fr/nsdc/
} 
the TV cameras on Mariner 9, the Visual Imaging Subsystem (VIS) on Viking 1 and 2 (Duxbury \& Callahan 1988, 1989), the Videospectrometric System (VSK) on Phobos 2 (Kolyuka et al. 1991), the ONC on MRO (Jacobson 2012, priv. comm.), and the Super Resolution Channel (SRC) on Mars Express (MEX; Oberst et al. 2006; Willner et al. 2008; Pasewaldt et al. 2012). In addition, ranging measurements to Phobos and observations of its shadow transiting the instrument's footprint on Mars' surface have been carried out by the Mars Orbiter Laser Altimeter (MOLA) on Mars Global Surveyor (MGS; Banerdt \& Neumann 1999; Bills et al. 2005). Phobos has also been observed during daytime - transiting across the solar disk - and during nighttime by the cameras on the Mars Exploration Rovers (Bell et al. 2005; Dehant et al. 2006) and recently by the Mast Camera (Mastcam) on the Mars Science Laboratory (MSL; Lemmon et al. 2013).

Essentially, two different techniques have been used to measure image positions of the disk-resolved Martian moons: Oberst et al. (2006) observed Phobos' and Deimos' center of figures (COFs) by fitting the limbs of triaxial ellipsoids to the observed limbs. Willner et al. (2008) used the Phobos control point network to determine the Phobos COF in SRC images (Kolyuka et al. 1991). Pasewaldt et al. (2012) also applied the limb-fit method on images of Deimos, albeit based on 3D shape models. In this paper, we compare the results of the two methods for Phobos images and define the conditions that need to be met to apply them.

This paper reports on astrometric observations from April 2008 to August 2011, when the MEX spacecraft (S/C) entered safe mode due to a failure in the onboard data storage system (SSMM). Since May 2013, the SRC has been operational again.

\section{Mars Express mission and HRSC/SRC camera}

\subsection{Mars Express}

In early June 2003, MEX was launched from the Baikonur Cosmodrome in Kazakhstan and was on Mars orbit about six months later at the end of December.

Since then, the $\mathrm{S} / \mathrm{C}$ is in a highly elliptical (pericenter $330 \mathrm{~km}$, apocenter $10530 \mathrm{~km})$, nearly polar $(86.9 \mathrm{deg})$ trajectory around Mars that reaches well beyond the Phobos orbit. Its orbital period (about seven hours) is similar to that of the Martian moon ${ }^{2}$. As a result of Mars' oblateness, the MEX orbit is subject to apsidal and nodal precession with its longitude of ascending node moving westwards (opposite to the Phobos motion). This enables regular and repeated encounters with the satellite approximately every six months (Witasse et al. 2014).

Mars Express regularly performs flybys of Phobos. Images taken during these close approaches have been used to create a control-point network (Willner et al. 2010), a 3D shape model (Willner et al. 2010, 2014), and a topographic image atlas (Wählisch et al. 2010, 2014). The control-point network and the 3D shape model were used for the astrometric measurement methods applied in this paper.

From April 2008 to August 2011, Phobos was observed between 0 and $90 \mathrm{deg}$ and between 180 and $360 \mathrm{deg}$ mean anomaly. More than three quarters of our observations lie between \pm 90 deg mean anomaly around the Phobos (evolving) periapsis (see Fig. 1).

\footnotetext{
2 MEX Fact Sheet: http://sci.esa.int/mars-express/ 47364-fact-sheet/
}

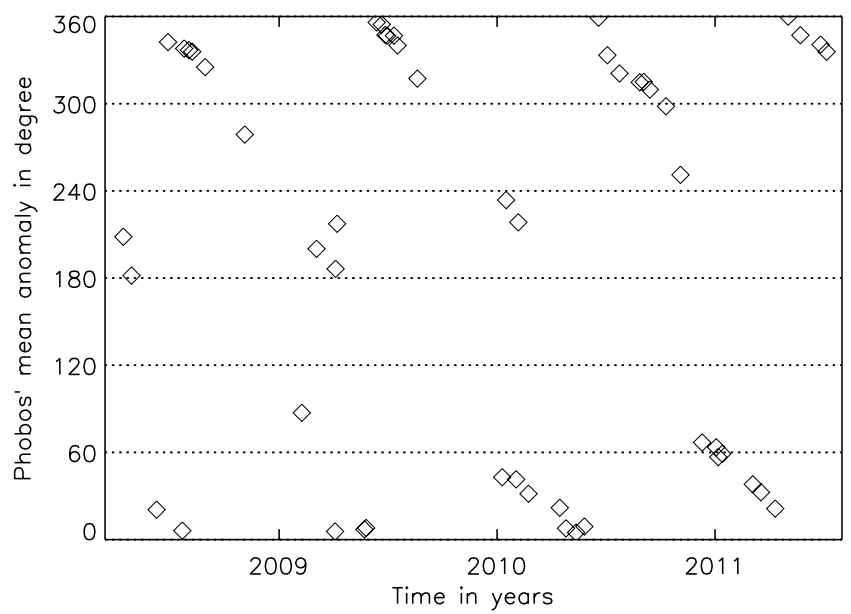

Fig. 1. Phobos' mean anomaly in degrees at mid-time of image sequence; each diamond stands for one sequence of eight images.

\section{2. $H R S C / S R C$ camera}

Mars Express carries seven scientific instruments, including the HRSC/SRC camera. The High Resolution Stereo Camera (HRSC) is a push-broom scanner comprising multiple CCD lines mounted in parallel on one focal plane for simultaneous highresolution stereo, multicolor, and multiphase imaging of the Martian surface (Jaumann et al. 2007). The SRC is a $1 \mathrm{~K} \times 1 \mathrm{~K}$ framing CCD that provides images embedded within the HRSC swath with a resolution that is nominally five times higher than the HRSC's resolving power (Oberst et al. 2008).

\subsection{SRC systematic processing}

Systematic processing of SRC images is carried out at the DLR Berlin, Germany. Processing Level 1 includes sorting of data from different sensors, composing of images from packets and frames, calculation of acquisition times, and removing offset, electronic, and dark pixels. The following radiometric calibration involves dark-frame subtraction, flat-field correction, and marking of blemish pixels (Processing Level 2). The final dimensions of SRC Level-2 images are 1008 samples by 1018 lines.

\subsection{SRC camera calibration}

A first in-flight calibration has been performed at the beginning of the mission using images of the Earth, Moon, and stars (Oberst et al. 2008). Particularly, the SRC's mounting alignment angles, calibrated focal length, and point-spread function (PSF) have been estimated (see Table 1 ).

More recently, Duxbury (2012, unpublished paper) updated these parameters using among other things image sequences of the Pleiades and an advanced method for star position measurement. From this, a new PSF was derived from a number of selected star images and was applied in a Richardson-Lucy image restoration process. Star locations have been measured in deblurred images fitting 2D parabolas and evaluated based on their signal-to-noise ratios $(\mathrm{S} / \mathrm{N})$. Owing to the low number of stars in the image corners, the two radial distortion coefficients could not be estimated with adequate statistical significance (Duxbury 2014, priv. comm.). For our astrometric reduction we used the new camera geometric parameters in combination with the mounting alignment angles. 
Table 1. SRC camera calibration parameters.

\begin{tabular}{lcrr}
\hline \hline Parameter & Unit & 1st In-flight & 2nd In-flight \\
\hline$f^{a}$ & {$[\mathrm{~mm}]$} & 988.60 & 984.76 \\
$1 / K^{b}$ & {$[\mathrm{~mm} / \mathrm{px}]$} & 0.009 & 0.009 \\
$s_{0}{ }^{c}$ & {$[\mathrm{px}]$} & 512.5 & 512.5 \\
$l_{0}{ }^{c}$ & {$[\mathrm{px}]$} & 512.5 & 512.5 \\
$\alpha_{1}{ }^{d}$ & {$\left[\mathrm{px} / \mathrm{mm}^{3}\right]$} & 0.0 & $-0.6644 \times 10^{-4}$ \\
$\alpha_{2}{ }^{d}$ & {$\left[\mathrm{px} / \mathrm{mm}^{5}\right]$} & 0.0 & $0.3087 \times 10^{-5}$ \\
$\Theta_{X}{ }^{e}$ & {$[\mathrm{deg}]$} & -0.0735 & -0.084154 \\
$\Theta_{Y}{ }^{e}$ & {$[\mathrm{deg}]$} & -0.0301 & -0.038531 \\
$\Theta_{Z}{ }^{e}$ & {$[\mathrm{deg}]$} & 90.0 & 90.038 \\
\hline
\end{tabular}

Notes. ${ }^{(a)}$ Calibrated focal length. ${ }^{(b)}$ Pixel size. ${ }^{(c)}$ CCD center. ${ }^{(d)}$ Radial distortion parameters. ${ }^{(e)}$ Mounting alignment angles.

References. (1) Duxbury (2012, unpublished paper); (2) Oberst et al. (2008).

\subsection{SRC image restoration}

SRC images are known to suffer from noise, blur and ghost features (for details, see Oberst et al. 2008). To improve our positional observations, we used images that were restored using a Richardson-Lucy deconvolution. This algorithm describes an iterative procedure for recovering a latent image that has been blurred by a known PSF (Michael \& Neukum 2009). Motion smear was considered by smearing the PSF over a number of pixels in the direction of Phobos' motion (the sample direction). As a result, the $\mathrm{S} / \mathrm{N}$ of stars were improved, and limbs and crater rims became sharper and better to discern. For our astrometric measurements, we used restored images.

\section{Data and methods}

\subsection{Image sequences}

SRC images of Phobos are planned whenever the object's distance is between 5500 and $100 \mathrm{~km}$ and the pointing can be chosen such that at least one star of magnitude 8.0 is inside the field of view (FOV). At a distance of $5500 \mathrm{~km}$, the image resolution is still suitable for topographic mapping and at distances below $100 \mathrm{~km}$ the pixel dwell time becomes so short that either the exposure time is chosen too long and image smear occurs, or the exposure time is chosen appropriately and the $\mathrm{S} / \mathrm{N}$ becomes too low.

During most Phobos flybys, the SRC is pointed at a fixed spot on the celestial sphere (inertial pointing), awaiting the satellite to cross its FOV. Within about $30 \mathrm{~s}$, a sequence of eight images is taken, of which the first and last images are longtime exposures made with the aim to detect the weak signal of background stars. Exposure durations of the images in between are adjusted to the brighter Phobos surface. We chose 52 of 74 Phobos flybys that offered the possibility of pointing verification at the beginning and end of the sequence (see Table 2).

\subsection{MEX orbit data}

Nominal MEX positions at SRC image mid-times can be retrieved from the European Space Operations Centre (ESOC) navigation orbits. According to ESOC's flight dynamics team, these reconstructed $\mathrm{S} / \mathrm{C}$ ephemerides have an uncertainty of
Table 2. MEX S/C flybys and Phobos mean anomalies.

\begin{tabular}{|c|c|c|c|c|}
\hline Orbit & $\begin{array}{l}\text { Image time } \\
\text { [UTC] }\end{array}$ & $\begin{array}{l}\mathrm{MA}^{a} \\
{[\mathrm{deg}]}\end{array}$ & $\begin{array}{r}\text { Range } \\
{[\mathrm{km}]}\end{array}$ & $\begin{array}{l}\mathrm{BGSD}^{b} \\
{[\mathrm{~km} / \mathrm{px}]}\end{array}$ \\
\hline 5504 & 2008-04-15T04:22 & 208.4 & 2325 & 0.021 \\
\hline 5552 & 2008-04-28T21:03 & 181.7 & 5134 & 0.047 \\
\hline 5699 & 2008-06-09T20:20 & 20.6 & 3859 & 0.035 \\
\hline 5766 & $2008-06-28 T 22: 55$ & 342.4 & 2486 & 0.023 \\
\hline 5850 & 2008-07-22T21:41 & 6.2 & 4490 & 0.041 \\
\hline 5861 & 2008-07-26T01:39 & 338.0 & 2300 & 0.021 \\
\hline 5889 & 2008-08-03T01:02 & 336.8 & 667 & 0.006 \\
\hline 5908 & 2008-08-08T11:11 & 335.8 & 1036 & 0.009 \\
\hline 5984 & 2008-08-30T03:37 & 325.2 & 2377 & 0.022 \\
\hline 6217 & 2008-11-04T11:14 & 278.8 & 4849 & 0.044 \\
\hline 6551 & 2009-02-08T00:15 & 87.3 & 5547 & 0.051 \\
\hline 6637 & 2009-03-04T16:11 & 200.2 & 5299 & 0.048 \\
\hline 6745 & 2009-04-04T18:26 & 5.6 & 5312 & 0.049 \\
\hline 6748 & 2009-04-05T13:35 & 186.3 & 5318 & 0.049 \\
\hline 6757 & 2009-04-08T03:29 & 217.4 & 4495 & 0.041 \\
\hline 6916 & 2009-05-23T21:36 & 7.0 & 529 & 0.005 \\
\hline 6926 & 2009-05-26T18:32 & 8.0 & 971 & 0.009 \\
\hline 6987 & 2009-06-13Т07:24 & 355.9 & 1754 & 0.016 \\
\hline 7017 & 2009-06-21T22:06 & 354.5 & 2893 & 0.026 \\
\hline 7038 & 2009-06-27T23:25 & 347.3 & 2756 & 0.025 \\
\hline 7048 & 2009-06-30T20:19 & 346.8 & 2843 & 0.026 \\
\hline 7088 & 2009-07-12T07:58 & 346.9 & 4015 & 0.037 \\
\hline 7109 & 2009-07-18T09:18 & 340.1 & 3875 & 0.035 \\
\hline 7225 & 2009-08-20T13:06 & 317.3 & 5104 & 0.047 \\
\hline 7719 & 2010-01-09T14:15 & 43.0 & 5363 & 0.049 \\
\hline 7742 & 2010-01-16T03:24 & 233.7 & 4416 & 0.040 \\
\hline 7800 & 2010-02-01T21:10 & 41.5 & 4548 & 0.042 \\
\hline 7813 & 2010-02-05T13:29 & 235.6 & 8216 & 0.075 \\
\hline 7872 & 2010-02-22T14:39 & 31.6 & 3904 & 0.036 \\
\hline 8052 & 2010-04-15T22:09 & 22.0 & 2542 & 0.023 \\
\hline 8088 & 2010-04-26T10:31 & 7.9 & 3996 & 0.037 \\
\hline 8146 & 2010-05-13T08:15 & 5.0 & 4202 & 0.038 \\
\hline 8193 & 2010-05-27T01:35 & 9.1 & 4933 & 0.045 \\
\hline 8276 & 2010-06-19T23:38 & 359.2 & 5486 & 0.050 \\
\hline 8326 & 2010-07-04T15:17 & 333.4 & 4193 & 0.038 \\
\hline 8396 & 2010-07-25T01:02 & 320.8 & 3469 & 0.032 \\
\hline 8512 & 2010-08-27T20:31 & 314.7 & 828 & 0.008 \\
\hline 8535 & 2010-09-03T13:19 & 315.0 & 785 & 0.007 \\
\hline 8570 & 2010-09-13T18:13 & 309.7 & 1177 & 0.011 \\
\hline 8663 & 2010-10-10T20:49 & 298.2 & 2743 & 0.025 \\
\hline 8746 & 2010-11-04T01:43 & 251.0 & 5585 & 0.051 \\
\hline 8870 & 2010-12-10T06:44 & 66.9 & 3642 & 0.033 \\
\hline 8951 & 2011-01-02T21:16 & 63.6 & 1205 & 0.011 \\
\hline 8963 & 2011-01-06T09:21 & 56.8 & 2394 & 0.022 \\
\hline 8986 & 2011-01-13Т02:11 & 59.1 & 1182 & 0.011 \\
\hline 9161 & 2011-03-05T02:50 & 38.1 & 3385 & 0.031 \\
\hline 9208 & 2011-03-18T19:57 & 32.6 & 4159 & 0.038 \\
\hline 9290 & 2011-04-11T17:58 & 21.4 & 5357 & 0.049 \\
\hline 9365 & 2011-05-03T10:09 & 359.9 & 4541 & 0.042 \\
\hline 9435 & 2011-05-23T19:55 & 347.1 & 3719 & 0.034 \\
\hline 9551 & 2011-06-26T15:23 & 340.7 & 885 & 0.008 \\
\hline 9586 & 2011-07-06T20:18 & 335.7 & 1050 & 0.010 \\
\hline
\end{tabular}

Notes. (a) Column MA gives the mean anomaly of Phobos. (b) Column BGSD gives the best ground sampling distance of the SRC image.

about $\pm 224 \mathrm{~m}$ (T. Morley 2011, priv. comm.). In parallel, MEX accurate orbits have been developed at the Royal 
Observatory of Belgium (ROB) especially for the purposes of Martian moon orbit modeling and Phobos mass determination. From processing Doppler and range radio tracking data from the Mars Radio Science (MaRS) experiment with a functionally enhanced version of the Géodésie par Intégration Numérique Simultanée (GINS) software, the average accuracy of these orbits is estimated to be about $\pm 20-25 \mathrm{~m}$ (Rosenblatt et al. 2008).

\subsection{MEX attitude data and pointing verification}

While S/C pointing data are nominally provided by ESOC (with nominal errors of $\pm 0.01 \mathrm{deg}$, Pischel \& Zegers 2009), we also used attitude data that were specifically generated at the European Space Astronomy Centre (ESAC), based on data from the MEX star sensors. Unfortunately, image sequences from orbit 7492 (Phobos and Deimos) and orbit 11964 (Pleiades) show damped harmonic oscillations around the $y$-axis of the $\mathrm{S} / \mathrm{C}$ (along the CCD $y$-direction) that are not represented in this reconstituted attitude data. Our analysis revealed two interfering oscillations with similar periods of about $8 \mathrm{~s}$, probably due to vibrations in $\mathrm{S} / \mathrm{C}$ structures subsequent to attitude maneuvers.

Therefore, we maintained procedures developed in our previous analyses (Willner et al. 2008; Pasewaldt et al. 2012), and verified and improved the camera pointing by using background stars with accurately known coordinates in the inertial reference frame (ICRF). We used Tycho-2 catalog data (Høg et al. 2000), which were corrected for proper motion, parallax, the relativistic deflection of light in the Sun's gravitational field, and stellar aberration (Kaplan et al. 1989).

By comparing the positions of predicted and observed stars in the image, we obtained differences in sample and line that represent our pointing correction. Deviations from the nominal pointing were determined for the first and last image of a sequence and were linearly interpolated in between. Because of the low number of stars per long-time exposure, image rotations around the camera boresight were not estimated. On the other hand, rotation angles derived in our control point analysis are very small, so that we neglected these at our level of accuracy. Star centers were measured interactively in restored images with an estimated accuracy of about one pixel. In the first and last images of each sequence, we measured at least one, on average one or two, and a maximum number of eight stars. Two image sequences with continuous observations of one background star were available.

Figures 2 and 3 show a comparison of the pointing corrections derived for ESOC-predicted and ESAC-reconstituted attitude data. The line offsets for reconstituted attitude data are slightly shifted from negative to positive values, becoming more normally distributed.

\subsection{Phobos COM measurements}

We used two similar approaches to determine the position of the disk-resolved Phobos, namely measuring control points and the limb-fit technique. While the former is based on Phobos' control-point network, the latter is founded on Phobos' gridded shape model. In the implementation of these methods we used the latest versions of both models by Willner et al. (2010, 2014). The coordinates of control points are believed to be given with respect to the Phobos center of mass (COM) as defined by the MAR080 ephemeris. Uncertainties in the camera positions and

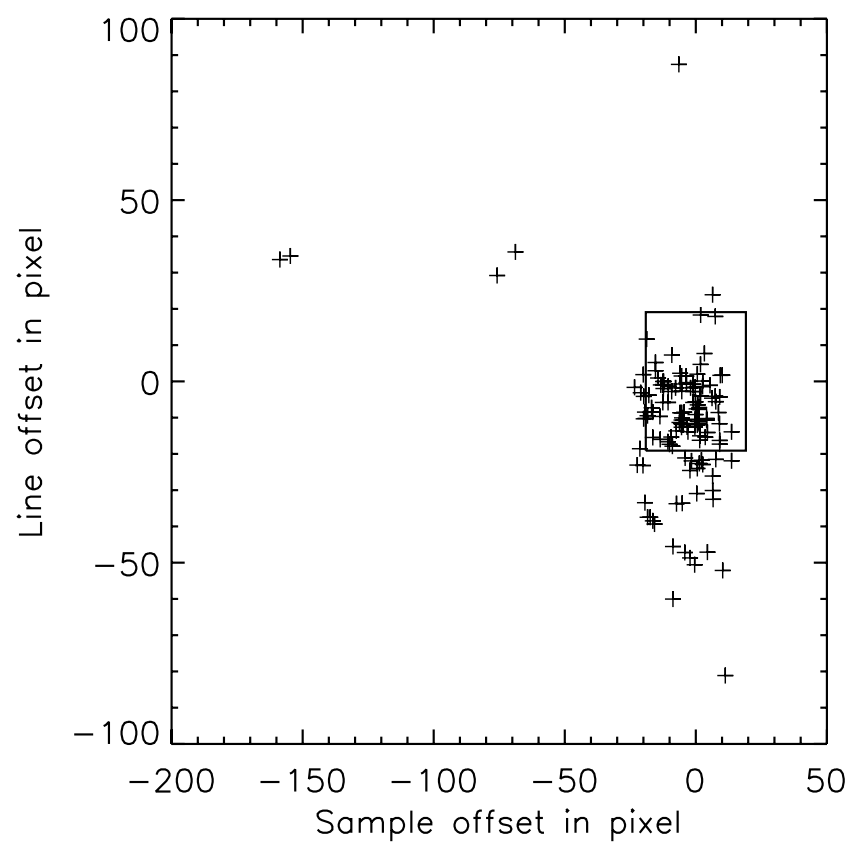

Fig. 2. SRC pointing corrections relating to ESOC-predicted attitude determined for the first and last images of each sequence in pixels. The rectangular box measures $38 \times 38$ pixels $^{2}$ in size and corresponds to the accuracy of predicted $C$ kernels of about $\pm 0.01 \mathrm{deg}$.

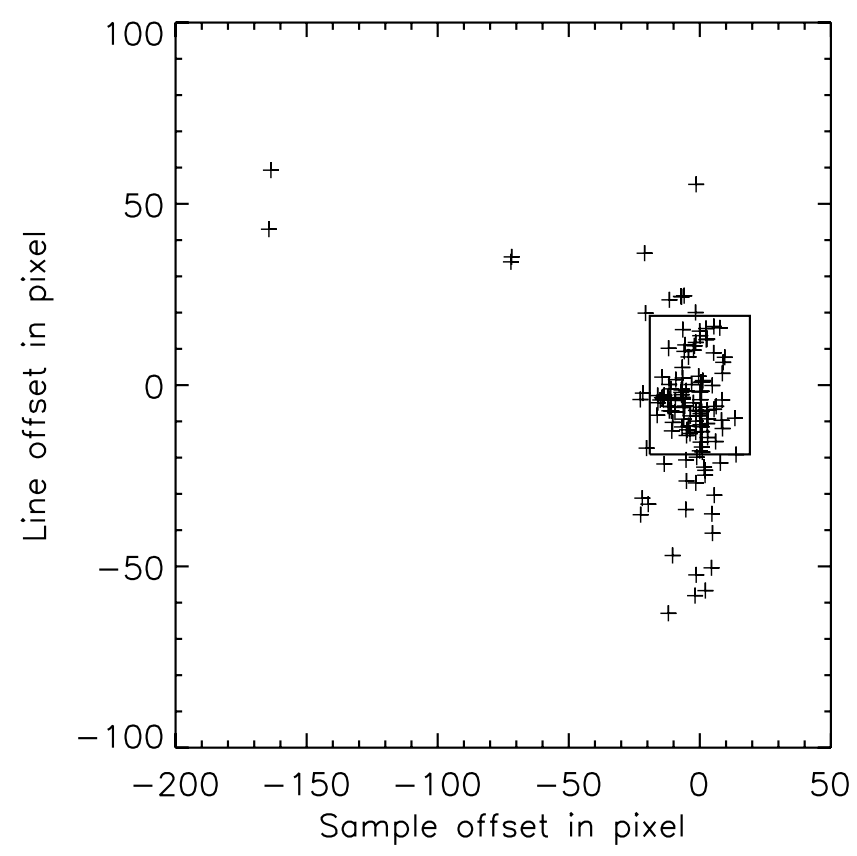

Fig. 3. SRC pointing corrections relating to ESAC-measured attitude determined for the first and last images of each sequence in pixels. The rectangular box measures $38 \times 38$ pixels $^{2}$ in size and corresponds to the accuracy of predicted $\mathrm{C}$ kernels of about $\pm 0.01 \mathrm{deg}$.

pointings for images, in which the control points were measured, were minimized. At the same time, the network's COM still contains the uncertainties in Phobos' positions. Therefore, we also refer to it in the following discussion as COF. The gridded shape model was co-registered to the control-point network and gives the positions of surface points relative to a COF. Offsets between control points and corresponding surface points are only up to $11 \mathrm{~m}$. 

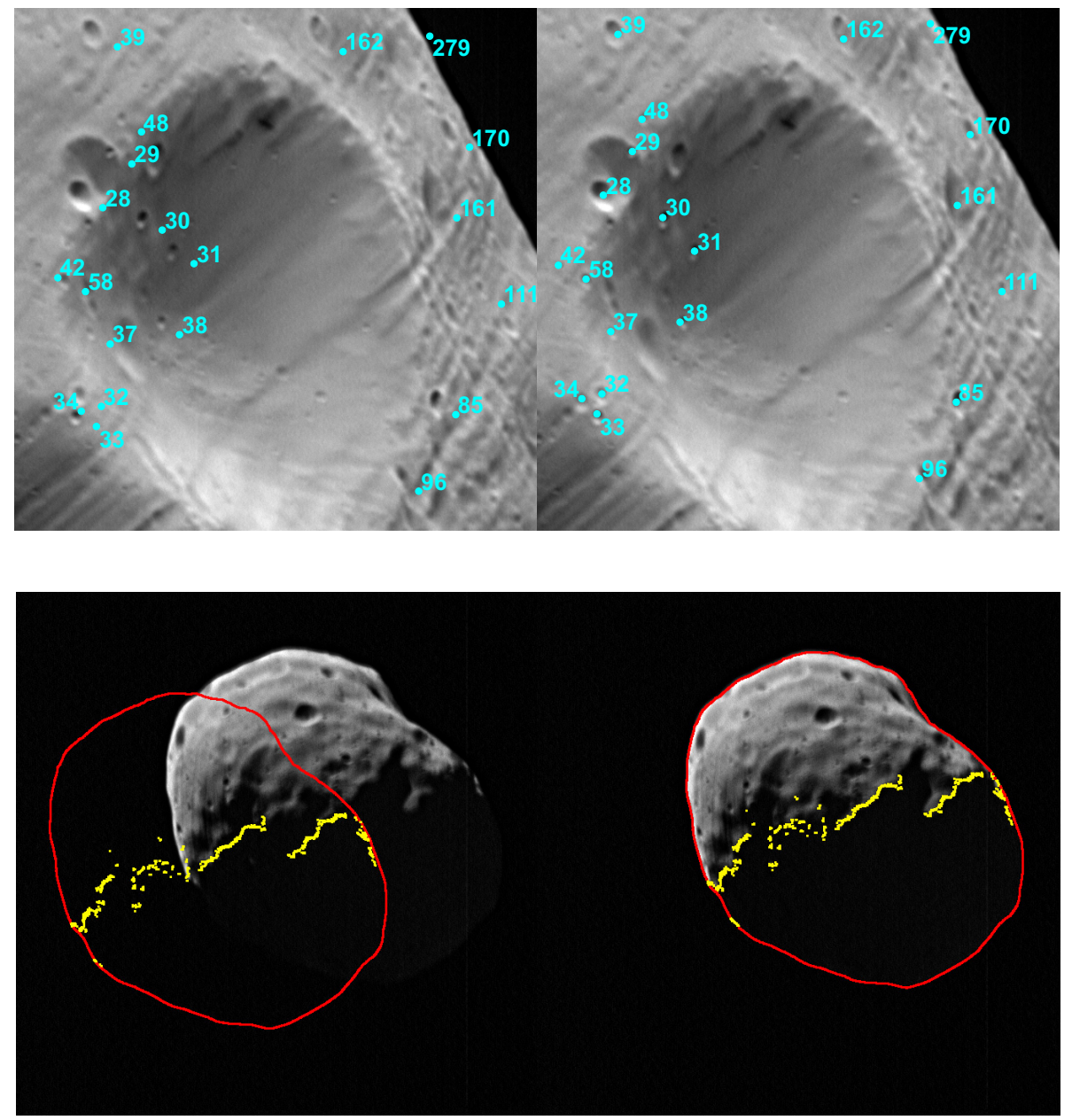

Fig. 4. Control point measurements: 1) prediction of control points; 2) visual identification of corresponding craters; 3 ) manual measurement of the centers of crater bottoms; 4) derivation of parameters transforming from predicted to observed control points; 5) application of transformation parameters on the predicted COF.

Fig. 5. Limb fit procedure: prediction of limb points; 2) manual fitting to visible limb; 3) detection of corresponding limb points using the predicted limb as starting point; 4) manual removal of wrongly recognized limb points; 5) fitting of predicted to observed limb points.

The common aim of the two methods is to measure the position of Phobos' COM. Because the true Phobos COM may not be known, we assumed that possible deviations between our models' COFs and Phobos' COM are small. To obtain a positional observation we predicted, identified, and measured control and limb points, derived parameters transforming from predicted to observed control and limb points, and applied these on the predicted Phobos COM.

First, we computed the apparent positions of control and limb points as seen from MEX and projected them onto the image plane. These computations were performed based on representations of Phobos' orbit, rotation, and shape by using the SPICE toolkit (Acton 1996). Then, we identified and measured the positions of corresponding image points by evaluating the pixel values. For the purpose of control-point observations, this step was performed manually using an overlay that shows point positions and identifiers (see Fig. 4). In the limb-fit approach, this task was achieved using a semi-automatic workflow (see Fig. 5; for details, see Pasewaldt et al. 2012).

The parameters for transforming from predicted to observed control and limb points were determined by using a least-squares method. As functional model, a four-parameter similarity transformation was chosen. Incorrectly identified control points were removed automatically based on the method of standardized residuals (Baarda 1968),

$$
\left[\begin{array}{l}
x_{\mathrm{o}} \\
y_{\mathrm{o}}
\end{array}\right]=s *\left[\begin{array}{cc}
\cos (\alpha) & \sin (\alpha) \\
-\sin (\alpha) & \cos (\alpha)
\end{array}\right] \times\left[\begin{array}{l}
x_{\mathrm{p}} \\
y_{\mathrm{p}}
\end{array}\right]+\left[\begin{array}{l}
x_{\mathrm{t}} \\
y_{\mathrm{t}}
\end{array}\right]
$$

where $x_{0}, y_{\mathrm{o}}$ and $x_{\mathrm{p}}, y_{\mathrm{p}}$ represent the coordinates of the observed and predicted point, $s$ is a scale factor, $\alpha$ is the rotation angle around the camera boresight (twist angle), and $x_{\mathrm{t}}$ and $y_{\mathrm{t}}$ are translation parameters corresponding to the elevation and crosselevation rotations (or equivalent shifts in S/C position).

To apply both approaches to SRC images, the visibility of the entire Phobos disk has been advantageous. While at low phase angles a larger portion of the limb is detectable, at medium phase angles, craters are better discernible because their rims cast shadows. Limb-fits can be performed in images of lower spatial resolution with unresolved craters, and control point measurements can be implemented at large image scales without visible limb. Special effects such as image smear, blur and ghost features simply decrease the image resolution. Pixel oversaturation due to long exposure times may also impair the limb fitting, but especially the measurement of control points. However, a visual evaluation of the transformed control points looking at the limb and vice versa increased the consistency between the two data sets.

\section{Results and discussion}

Altogether, we measured in 158 SRC images positions of Phobos. Therefore, we performed 130 control-point and 131 limb-fit observations. On 103 SRC images we applied both methods. Our directions to Phobos are given in S/C-centered right ascension and declination coordinates in the International Celestial Reference Frame (ICRF). 
Table 3. Sample of the results of our astrometric reduction.

\begin{tabular}{lccccccccc}
\hline \hline Img. name & $\begin{array}{c}\text { Img. mid-time } \\
{[\mathrm{UTC}]}\end{array}$ & $M^{a}$ & $\begin{array}{c}\mathrm{RA}_{\mathrm{PHO}} \\
{[\mathrm{deg}]}\end{array}$ & $\begin{array}{c}\mathrm{Dec} \text { PHO } \\
{[\mathrm{deg}]}\end{array}$ & $\begin{array}{c}\sigma_{\mathrm{PHO}} \\
{[\mathrm{deg}]}\end{array}$ & $N^{b}$ & $\begin{array}{c}X_{\mathrm{MEX}} \\
{[\mathrm{km}]}\end{array}$ & $\begin{array}{c}Y_{\mathrm{MEX}} \\
{[\mathrm{km}]}\end{array}$ & $\begin{array}{c}Z_{\mathrm{MEX}} \\
{[\mathrm{km}]}\end{array}$ \\
\hline h5504_0004 & 2008-04-15T04:22:13.755Z & CP & 145.0878 & 35.3969 & 0.0056 & 7 & -5866.42 & 1373.32 & 4057.15 \\
h5504_0005 & 2008-04-15T04:22:18.115Z & CP & 145.3555 & 35.1276 & 0.0056 & 5 & -5857.20 & 1367.76 & 4061.01 \\
h5552_0004 & 2008-04-28T21:03:41.149Z & CP & 111.2926 & 17.9497 & 0.0053 & 8 & -3856.85 & 503.09 & 4192.81 \\
h5552_0005 & 2008-04-28T21:03:45.509Z & CP & 111.4968 & 17.9176 & 0.0053 & 8 & -3844.94 & 496.53 & 4195.37 \\
h5552_0006 & 2008-04-28T21:03:49.869Z & CP & 111.6899 & 17.8489 & 0.0053 & 8 & -3833.01 & 489.97 & 4197.91 \\
h9551_0007 & 2011-06-26T15:23:49.532Z & LF & 28.2579 & -51.6005 & 0.0147 & 8 & 2069.66 & 8207.38 & 3377.03 \\
h9586_0003 & 2011-07-06T20:18:08.066Z & LF & 42.7001 & -21.9013 & 0.0129 & 6 & 1970.16 & 7795.11 & 2994.03 \\
h9586_0004 & 2011-07-06T20:18:10.791Z & LF & 43.1984 & -21.9156 & 0.0129 & 15 & 1971.35 & 7789.79 & 2996.93 \\
h9586_0005 & 2011-07-06T20:18:13.517Z & LF & 43.7074 & -21.9077 & 0.0130 & 19 & 1972.53 & 7784.47 & 2999.84 \\
h9586_0006 & 2011-07-06T20:18:16.242Z & LF & 44.2095 & -21.9044 & 0.0130 & 7 & 1973.72 & 7779.14 & 3002.74 \\
\hline
\end{tabular}

Notes. ${ }^{(a)}$ Column $M$ gives the measurement method. ${ }^{(b)}$ Column $N$ gives the number of control points or the percentage of limb.

The results are provided in tabular form including image name and mid-time in Coordinated Universal Time (UTC), equatorial coordinates of Phobos in the S/C-centered ICRF, the associated one-sigma uncertainty, and rectangular coordinates of MEX in the Mars-centered ICRF (see Table 3). Additionally, we state the technique used $(\mathrm{CP} / \mathrm{LF})$ and the number of observed control points or the percentage of measured limb points. The latter describes the portion of Phobos' apparent limb that is illuminated and inside the SRC FOV.

We provide two data sets, each including both the controlpoint and the limb-fit measurements (103 observations are supplied twice within both sets). The first set of positions is corrected for pointing offsets with respect to ESOC-predicted attitude data with the S/C positions retrieved from ESOC MEX navigation orbits. In the second set of positions we applied corrections relative to ESAC-measured pointing data with S/C positions based on ROB MEX accurate orbits (see Tables A.1 and A.2).

\subsection{Sources of errors and prediction of uncertainties}

Sources of error of our S/C-based astrometric observations are the position of the MEX S/C, the pointing of the SRC camera, and the measurement of Phobos' position.

The measurement uncertainty $\left(\sigma_{\text {cof }}\right)$ was derived from the uncertainty in parameters transforming predicted to observed control or limb points. We determined the positions of control and limb points in the image with accuracies of \pm 1.0 and 0.5 pixel, respectively. As described above, the phase angle plays an important role regarding the number and distribution of control points or the length of the available limb, but its effects are difficult to quantify. Hence, we did not include it in our uncertainty estimation. Instead, we provide the number of observed control points or the percentage of measured limb points that were used to determine Phobos' position.

We typically estimated the pointing uncertainty $\left(\sigma_{\mathrm{pnt}}\right)$ as the sum of the accuracy in offset measurement and half of the range between the pointing offsets of the first and last images in a sequence (interpolation uncertainty). When Phobos is observed in combination with a star, however, the second term was ignored. The accuracy of star position observations is estimated to be about \pm 1.0 pixel, which means that it is at least two orders of magnitude larger than the accuracy of our star position predictions. Because we have two pointing offsets for most sequences, the only possible assumption for interpolation is a linear pointing drift. If $\mathrm{S} / \mathrm{C}$ jitter occurs subsequent to an attitude maneuver, our uncertainty estimate may be only appropriate if the first and last images were taken at times of positive and negative amplitudes. Otherwise, we may underestimate our pointing uncertainty.

Previously, we assumed an uncertainty in $\mathrm{S} / \mathrm{C}$ position $\left(\sigma_{\text {pos }}\right)$ of about $\pm 500 \mathrm{~m}$. Since the beginning of the mission, MEX tracking and reconstruction techniques have been improved by using shorter reaction wheel desaturation maneuvers and longer radio tracking data arcs (T. Morley 2011, priv. comm.). Because our observation period starts four years after the beginning of the mission, our observational accuracy may benefit from recent developments. Consequently, we adopted a smaller uncertainty in S/C position of about $\pm 224 \mathrm{~m}$,

$\sigma=\sqrt{\sigma_{\text {cof }}^{2}+\sigma_{\mathrm{pnt}}^{2}+\left(\arctan \left(\frac{\sigma_{\mathrm{pos}}}{d}\right)\right)^{2}}$,

where $\mathrm{d}$ is the distance between MEX and Phobos (Duxbury \& Callahan 1988).

Measurement uncertainties in our astrometric observations vary between 0.224 and $3.405 \mathrm{~km}$. The mean accuracy is $407 \pm$ $460 \mathrm{~m}$. The uncertainty in $\mathrm{S} / \mathrm{C}$ position contributes a constant large part to the total error budget. In about one fourth of all flybys that show a wide range of pointing offsets, the respective uncertainty exceeds this value several times (see Table 4). The largest uncertainty in camera pointing in orbit 6637 is with $3.397 \mathrm{~km}$ three times as high as the next lower values of 1.093 or $0.812 \mathrm{~km}$ in orbits 6557 and 6748 . The uncertainty in position observation represents the smallest kind of error with values up to $87 \mathrm{~m}$.

On average, uncertainty estimates of control-point measurements are smaller than those derived for limb-fit observations. The former exceed the latter only in few cases (see Fig. 6).

\subsection{Comparison between control-point and limb-fit observations}

To compare the positions derived with the two methods we calculated the distances between 103 corresponding observations. Depending on the distance to Phobos, an average distance of 2.4 pixels in image space is related to $79 \mathrm{~m}$ in object space. Observations from images h9586_0005 and h7225_0005 show the largest distances with 21.9 pixels $(\hat{=} 210 \mathrm{~m})$ and $\overline{245} \mathrm{~m}$ ( $\hat{=} 4.8$ pixels), respectively. These deviations can be explained by 


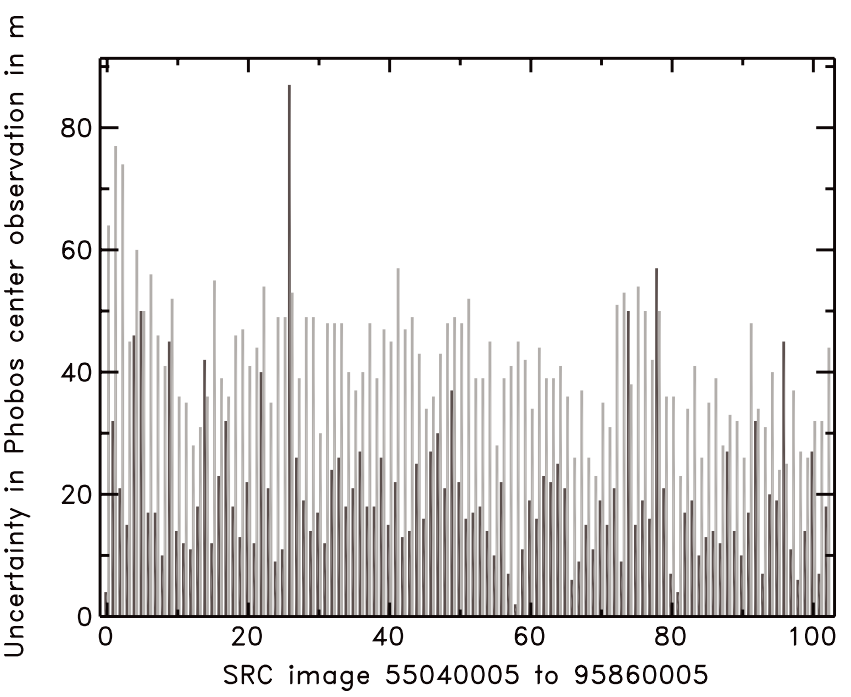

Fig. 6. Phobos positional uncertainties derived from control-point (dark grey bars) and limb-fit (light grey bars) observations in meters.

Table 4. Orbits during which the MEX pointing uncertainty exceeds the position uncertainty.

\begin{tabular}{lrr}
\hline \hline Orbit \# & $\begin{array}{r}\sigma_{\text {PNT }} \\
\text { pixel] }\end{array}$ & $\begin{array}{r}\sigma_{\text {PNT }} \\
{[\mathrm{km}]}\end{array}$ \\
\hline 7017 & 8.8 & 0.231 \\
7800 & 5.9 & 0.243 \\
7038 & 10.6 & 0.268 \\
7048 & 13.1 & 0.339 \\
7719 & 7.3 & 0.356 \\
7225 & 8.1 & 0.380 \\
5552 & 8.9 & 0.417 \\
8396 & 13.4 & 0.424 \\
8746 & 8.7 & 0.442 \\
9161 & 16.6 & 0.514 \\
7872 & 15.5 & 0.554 \\
8276 & 16.0 & 0.800 \\
6748 & 16.7 & 0.812 \\
6757 & 26.6 & 1.093 \\
6637 & 70.1 & 3.396 \\
\hline
\end{tabular}

the shape models we used and the different measurement quality (see Table 5 and Fig. 7).

As mentioned earlier, the gridded shape model was co-registered to the Phobos control point network. Offsets between corresponding control and surface points are only up to $11 \mathrm{~m}$. However, as examples of images h9586_0005 and 7225_0005 suggest, there may still be parts of the shape model that are only poorly constrained by the control-point network. The mean accuracy of control-point positions is $\pm 32 \mathrm{~m}$. The observation of control points of lower accuracies increases our uncertainty in position measurement. Simultaneously, the gridded shape model resolution is 100 by $100 \mathrm{~m}^{2}$, which means that local deviations from the true shape may occur.

For the purpose of measuring control points a low image resolution, shadowed crater bottoms, or observing craters nearby the limb may lead to inaccurate measurements or even point misidentifications. When detecting and fitting limb points, short limb curves of low curvature or local deviations of the predicted from the observed shape may limit the accuracy of our results.

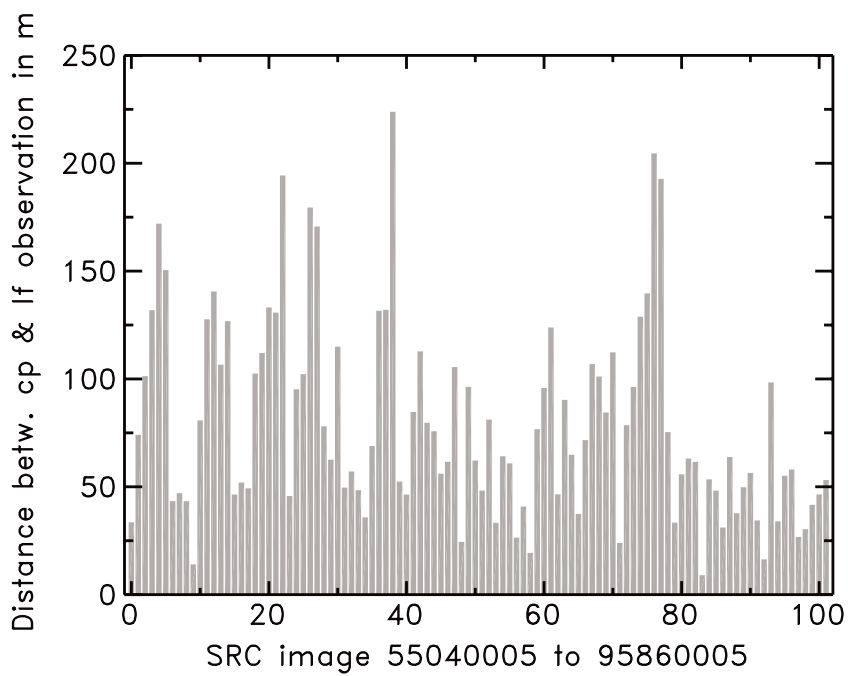

Fig. 7. Distances between control-point and limb-fit observations in meters.

Table 5. Euclidean distance between control-point and limb-fit observations.

\begin{tabular}{llrrrr}
\hline \hline & Unit & $x_{\min }$ & $x_{\max }$ & $\bar{x}$ & $\sigma$ \\
\hline $\mathrm{CP}^{a}-\mathrm{LF}^{b}$ Obs. & {$[\mathrm{px}]$} & 0.3 & 21.9 & 2.4 & 1.3 \\
$\mathrm{CP}^{a}-\mathrm{LF}^{b}$ Obs. & {$[\mathrm{m}]$} & 9.0 & 224.7 & 78.9 & 45.6 \\
\hline
\end{tabular}

Notes. ${ }^{(a)}$ Phobos position derived from control-point measurement. (b) Phobos position derived from limb-fit observation.

Table 6. Statistical quantities for $\mathrm{O}-\mathrm{C}$ residuals computed for controlpoint measurements with respect to the ROB and JPL orbit models.

\begin{tabular}{lllrrrr}
\hline \hline & Dir & Unit & $x_{\min }$ & $x_{\max }$ & $\bar{x}$ & $\sigma$ \\
\hline ROB & $\alpha \cos \delta$ & {$[\mathrm{km}]$} & -1.995 & 3.403 & 0.272 & 1.188 \\
& $\delta$ & {$[\mathrm{km}]$} & -2.354 & 3.372 & 0.047 & 1.096 \\
\hline JPL & $\alpha \cos \delta$ & {$[\mathrm{km}]$} & -1.938 & 1.027 & -0.217 & 0.450 \\
& $\delta$ & {$[\mathrm{km}]$} & -2.477 & 4.044 & 0.044 & 0.862 \\
\hline
\end{tabular}

Table 7. Statistical quantities for $\mathrm{O}-\mathrm{C}$ residuals computed for limb-fit observations with respect to the ROB and JPL orbit models.

\begin{tabular}{lllrrrr}
\hline \hline & Dir & Unit & $x_{\min }$ & $x_{\max }$ & $\bar{x}$ & $\sigma$ \\
\hline ROB & $\alpha \cos \delta$ & {$[\mathrm{km}]$} & -1.712 & 3.404 & 0.335 & 1.125 \\
& $\delta$ & {$[\mathrm{km}]$} & -2.320 & 4.373 & 0.223 & 1.071 \\
\hline JPL & $\alpha \cos \delta$ & {$[\mathrm{km}]$} & -1.870 & 1.028 & -0.186 & 0.445 \\
& $\delta$ & {$[\mathrm{km}]$} & -2.444 & 4.547 & 0.081 & 0.939 \\
\hline
\end{tabular}

\subsection{Comparison of astrometric residuals relating to orbit models}

We compared our results with the predictions from models to evaluate the effect of fitting these dynamical theories of Phobos' motion to our observations and to assess the quality of our uncertainty estimates. Therefore, we computed the O-C residuals for the following reference ephemerides: the earlier MarsSatV1_0 model developed at the ROB (Lainey et al. 2007) and the more recent JPL MAR085 theory (Jacobson 2010). Differences between the two models are listed in Table 8. A more detailed discussion can be found in Jacobson \& Lainey (2014). 
Table 8. Differences between ROB MarsSatV1_0 and JPL MAR085 orbit models.

\begin{tabular}{|c|c|c|}
\hline & ROB MarsSatV1_01 & $\begin{array}{l}\text { JPL } \\
\text { MAR085² }\end{array}$ \\
\hline $\begin{array}{l}\text { Martian gravity } \\
\text { field }\end{array}$ & $\begin{array}{l}\text { MGM1041C up to } \\
\text { degree } 10\end{array}$ & $\begin{array}{l}\text { MGS95J up to } \\
\text { degree } 8 \text { in zonal and } \\
\text { degree and order } 5 \text { in } \\
\text { tesseral harmonics }\end{array}$ \\
\hline $\begin{array}{l}\text { Mars orientation } \\
\text { model }\end{array}$ & IAU 2000 model & $\begin{array}{l}\text { Orientation model } \\
\text { with nutation } \\
\text { (Konopliv et al. } \\
\text { 2006) }\end{array}$ \\
\hline $\begin{array}{l}\text { Solar system } \\
\text { ephemerides }\end{array}$ & DE406 & DE421 \\
\hline $\begin{array}{l}\text { Phobos and } \\
\text { Deimos mass }\end{array}$ & $\begin{array}{l}\text { Adopted from } \\
\text { literature }\end{array}$ & $\begin{array}{l}\text { Estimated from } \mathrm{S} / \mathrm{C} \\
\text { tracking data }\end{array}$ \\
\hline Tidal interactions & $\begin{array}{l}\text { Phobos and Deimos } \\
\text { tides on Mars }\end{array}$ & Phobos tides on Mars \\
\hline $\begin{array}{l}\text { Phobos gravity } \\
\text { field }\end{array}$ & $\begin{array}{l}\mathrm{C} 20 \text { and C22 } \\
\text { coefficients included } \\
\text { and estimated }\end{array}$ & $\begin{array}{l}\mathrm{C} 20 \text { and C22 } \\
\text { coefficients included }\end{array}$ \\
\hline $\begin{array}{l}\text { Phobos libration } \\
\text { in longitude }\end{array}$ & Not yet included & $\begin{array}{l}\text { Included and Phobos } \\
\text { forced libration angle } \\
\text { estimated }\end{array}$ \\
\hline $\begin{array}{l}\text { Earth-based } \\
\text { observations }\end{array}$ & $\begin{array}{l}\text { Observations through } \\
2003\end{array}$ & $\begin{array}{l}\text { Plus observations } \\
\text { from } \mathrm{TMO}^{a} \text { and } \\
\mathrm{LNA}_{\mathrm{MCT}}{ }^{b}\end{array}$ \\
\hline $\begin{array}{l}\text { S/C-based } \\
\text { observations }\end{array}$ & $\begin{array}{l}\text { Observations through } \\
2005^{c}\end{array}$ & $\begin{array}{l}\text { Plus observations } \\
\text { from MRO and } \\
\text { MEX }^{d}\end{array}$ \\
\hline
\end{tabular}

Notes. ${ }^{(a)}$ Table Mountain Observatory. ${ }^{(b)}$ Laboratório Nacional de Astrofísica do Ministério da Ciência e Tecnologia. ${ }^{(c)}$ Including Oberst et al. (2006). ${ }^{(d)}$ Including Willner et al. (2008).

References. (1) Lainey et al. (2007); (2) Jacobson (2010).

Generally, our observations agree well with the predictions from the two models (see Tables 6 and 7). The average $\alpha \cos \delta$ residuals for the ROB model are opposite in sign and larger than those for the JPL model. The total difference along this direction is about $500 \mathrm{~m}$ for both types of measurements. In addition, the dispersion of $\alpha \cos \delta$ residuals is significantly larger here. While for control-point measurements the residuals in $\delta$ behave quite similar for both models, they vary more strongly for the limbfit observations. Differences between control-point and limb-fit measurements for the JPL model are considerably smaller than for the ROB model.

The observed differences in astrometric residuals between orbit models can be explained by the relevance and accuracy of observations and/or differences in modeling. At the same time, limb-fit observations seem to be more sensitive toward the dissimilarity between the two ephemerides, which may be due to the deviations between corresponding limb-fit and control-point observations and to the probable presence of outliers in the additional 28 limb-fit measurements. Marked extrema are also probable because of the occurrence of $\mathrm{S} / \mathrm{C}$ oscillations subsequent to attitude maneuvers.

The residual plots (see Figs. 8-11) show that the variations between image sequences can be explained by these sources of error, while deviations within an image sequence are probably due to the occasional presence of $\mathrm{S} / \mathrm{C}$ oscillations and the first increasing and then decreasing portions of the Phobos disk inside the SRC FOV.

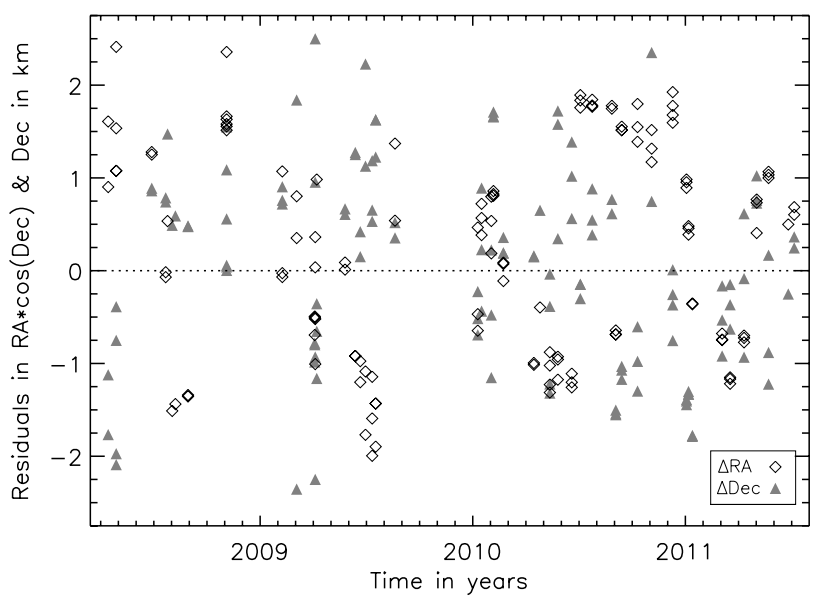

Fig. 8. O-C residuals of Phobos control-point observations relative to ROB (MarsSatV1_0) model in $\alpha \times \cos (\delta)$ (diamonds) and $\delta$ (triangles) in $\mathrm{km}$.

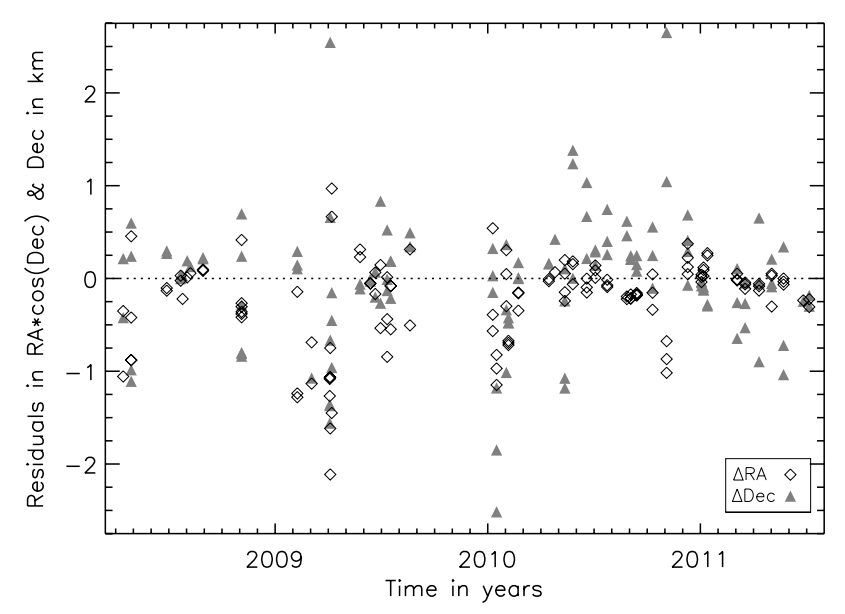

Fig. 9. O-C residuals of Phobos control-point observations relative to JPL (MAR085) model in $\alpha \times \cos (\delta)$ (diamonds) and $\delta$ (triangles) in $\mathrm{km}$.

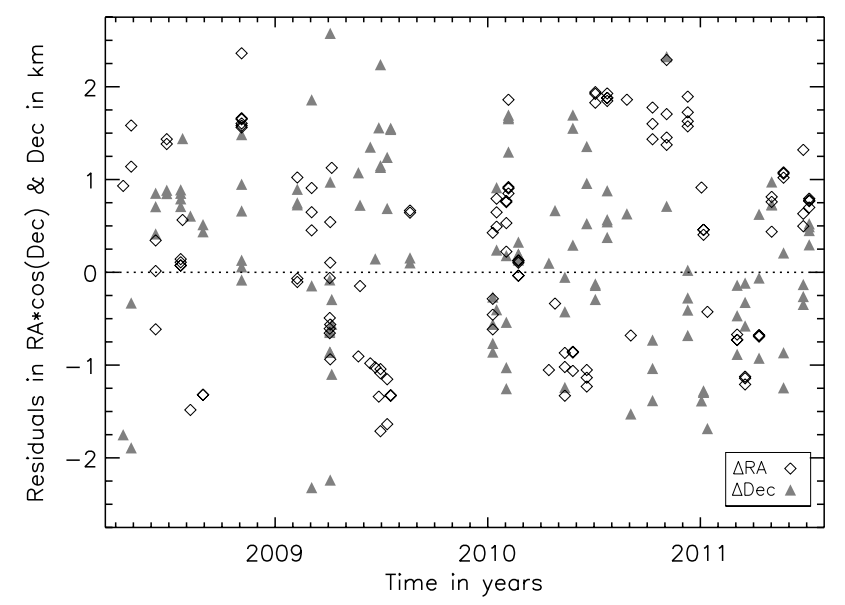

Fig. 10. O-C residuals of Phobos limb-fit observations relative to ROB (MarsSatV1_0) model in $\alpha \times \cos (\delta)$ (diamonds) and $\delta$ (triangles) in km.

\subsection{Comparison of observation reductions using different MEX orbit and attitude data}

Below we discuss the results of reducing our astrometric observations by using first MEX accurate orbits with ESOC predicted 


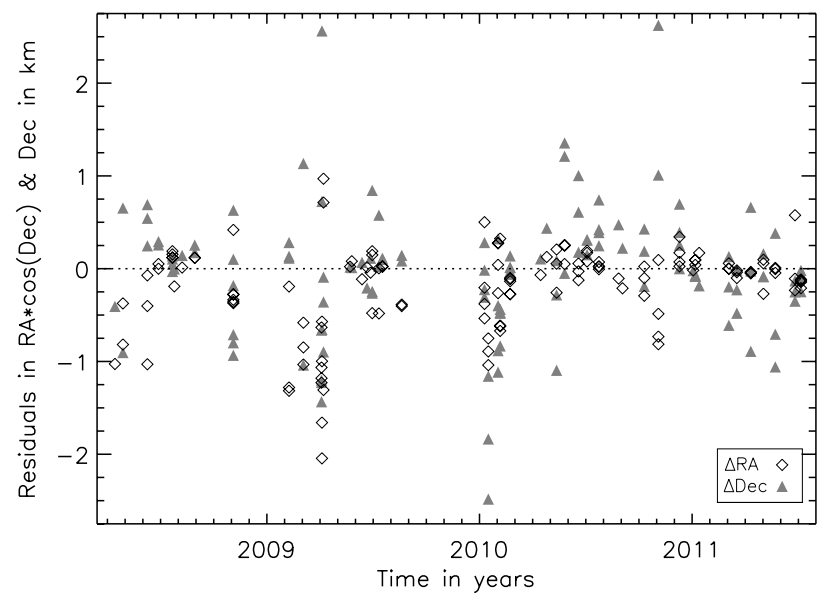

Fig. 11. O-C residuals of Phobos limb-fit observations relative to JPL (MAR085) model in $\alpha \times \cos (\delta)$ (diamonds) and $\delta$ (triangles) in $\mathrm{km}$.

Table 9. Available MEX orbit and attitude data.

\begin{tabular}{ll}
\hline \hline MEX orbit data & MEX attitude data \\
\hline ESOC reconstructed orbits & ESOC predicted attitude \\
ROB reconstructed orbits & ESAC reconstituted attitude \\
\hline
\end{tabular}

Table 10. Statistical quantities for $\mathrm{O}-\mathrm{C}$ residuals based on ROB MEX accurate orbits and ESOC MEX navigation orbits.

\begin{tabular}{lllcccc}
\hline \hline Orbit & Dir & Unit & $x_{\min }$ & $x_{\max }$ & $\bar{x}$ & $\sigma$ \\
\hline \multirow{2}{*}{ ESOC } & $\alpha \cos \delta$ & {$[\mathrm{km}]$} & -1.938 & 1.027 & -0.217 & 0.450 \\
& $\delta$ & {$[\mathrm{km}]$} & -2.477 & 4.044 & 0.044 & 0.862 \\
\hline ROB & $\alpha \cos \delta$ & {$[\mathrm{km}]$} & -1.934 & 1.033 & -0.234 & 0.455 \\
& $\delta$ & {$[\mathrm{km}]$} & -2.499 & 4.039 & 0.045 & 0.861 \\
\hline
\end{tabular}

Table 11. Statistical quantities for $\mathrm{O}-\mathrm{C}$ residuals based on ESOC-predicted attitude and ESAC-reconstituted attitude.

\begin{tabular}{llccccc}
\hline \hline Attitude & Dir & Unit & $x_{\min }$ & $x_{\max }$ & $\bar{x}$ & $\sigma$ \\
\hline ESOC & $\alpha \cos \delta$ & {$[\mathrm{km}]$} & -1.938 & 1.027 & -0.217 & 0.450 \\
& $\delta$ & {$[\mathrm{km}]$} & -2.477 & 4.044 & 0.044 & 0.862 \\
\hline \multirow{2}{*}{ ESAC } & $\alpha \cos \delta$ & {$[\mathrm{km}]$} & -1.946 & 0.862 & -0.240 & 0.456 \\
& $\delta$ & {$[\mathrm{km}]$} & -2.399 & 4.183 & 0.021 & 0.858 \\
\hline
\end{tabular}

attitude and then ESOC navigation orbits with ESAC reconstituted pointing (see Table 9). As reference data sets we chose our control-point observations in combination with the MAR085 ephemeris.

When our observations were reduced with ROB accurate orbits, the average and dispersion of $\alpha \cos \delta$ residuals increase slightly by 17 and $5 \mathrm{~m}$, respectively. Simultaneously, the residuals in $\delta$ remain almost constant (see Table 10).

Using the ESAC-reconstituted attitude without pointing control leads to a notable increase in astrometric residuals. Therefore, we derived a new set of pointing corrections. When we applied these offset values, we obtained an increase in $\alpha \cos \delta$ and a decrease in $\delta$ residuals, both by about $23 \mathrm{~m}$ (see Table 11).

Even though we were unable to achieve a significant decrease in astrometric residuals, using additional tracking data very likely provides more accurate trajectories and applying measured instead of predicted attitude data yields more correct $\mathrm{S} / \mathrm{C}$ orientations. The astrometric residuals we quoted are less a measure of quality for the used data than a quantity reflecting the consistency between our observations and the particular Phobos orbit. Hence, a possible explanation for observed discrepancies may be the contradiction between our data and MEX astrometric observations that are already included in the MAR085 ephemeris (Oberst et al. 2006; Willner et al. 2008). Consequently, our recommendation is to use only one of the two available orbit and/or attitude data sets to reduce all MEX astrometric observations ${ }^{3}$.

\section{Conclusions}

We reported on a set of 158 astrometric observations of Phobos with estimated uncertainties between 0.224 and $3.405 \mathrm{~km}$. These observations were derived from 130 control-point and 131 limbfit observations. The average offset between 103 corresponding measurements is about $79 \mathrm{~m}$ and can be explained by the difference between the shape models used and the feasible measurement accuracy.

We demonstrated that both measurement techniques are well-suited for determining Phobos positions. Control-point measurements can deliver accurate results. However, outliers can occur due to the operator's difficulties in identifying and measuring individual positions. In contrast, limb-fit observations are easier to automate and less prone to outliers. Short limbs usually restrict the accuracy of our results.

Our observations agree well with the JPL and ROB models, showing mean offsets of up to $335 \mathrm{~m}$. Average residuals for the ROB model are slightly larger than those for the JPL model. Even though the use of improved MEX orbit and attitude data did not decrease our astrometric residuals, it provides an alternative data set and partially confirmed the quality of our current reduction.

Larger residual variations between observations of different or the same image sequence(s) are explained by $\mathrm{S} / \mathrm{C}$ jitter occurring subsequent to attitude maneuvers. These oscillations are taking place around the S/C $y$-axis or along the CCD $y$-direction with approximate mean periods of $8 \mathrm{~s}$. We suspect that vibrations in $\mathrm{S} / \mathrm{C}$ structures are the reason for this.

Our observations can be used to maintain and update the current dynamical theories of the Martian satellites' motion.

Acknowledgements. Andreas Pasewaldt acknowledges support by the European Community's Seventh Framework Program (FP7/2007-2013) under grant agreement $\mathrm{N}^{\circ} 263466$ for the FP7-ESPaCE project. Jürgen Oberst and Anatoly Zubarev acknowledge the hospitality of MIIGAiK (Moscow State University for Geodesy and Cartography) and support by the Russian Science Foundation, project \#14-22-00197. The authors would like to thank the anonymous referees for their helpful comments and suggestions. Furthermore, they are grateful to Valery Lainey for various valuable discussions, Thomas C. Duxbury for detailed explanations of his work on SRC camera calibration, Robert A. Jacobson for data testing, and the FP7-ESPaCE project team.

\section{References}

Acton, C. H. 1996, Planet. Space Sci., 44, 65

Andert, T. P., Rosenblatt, P., Pätzold, M., et al. 2010, Geophys. Res. Lett., 37, 9202

Arlot, J.-E., \& Emelyanov, N. V. 2009, A\&A, 503, 631

Baarda, W. 1968, Publications on Geodesy - New Series, 2, 5

Banerdt, W. B., \& Neumann, G. A. 1999, Lunar and Planetary Science Conference, 30, 2021

Bell, J. F., Lemmon, M. T., Duxbury, T. C., et al. 2005, Nature, 436, 55

3 MEX SPICE SP kernels are available on:

ftp: //ssols01. esac . esa. int/pub/data/SPICE/MEX/kernels/ 
Bills, B. G., Neumann, G. A., Smith, D. E., \& Zuber, M. T. 2005, J. Geophys. Res. Planets, 110, 7004

Borderies, N., \& Yoder, C. F. 1990, A\&A, 233, 235

Dehant, V., Berthier, J., Bell, III, J., Lainey, V., \& Million, C. 2006, in AAS/Division for Planetary Sciences Meeting Abstracts \#38, BAAS, 38, 602 Duxbury, T. C., \& Callahan, J. D. 1988, A\&A, 201, 169

Duxbury, T. C., \& Callahan, J. D. 1989, A\&A, 216, 284

Duxbury, T. C. 2012, unpublished

Høg, E., Fabricius, C., Makarov, V. V., et al. 2000, A\&A, 355, L27

Jacobson, R. A. 2010, AJ, 139, 668

Jacobson, R. A., \& Lainey, V. 2014, Planet. Space Sci., 102, 35

Jaumann, R., Neukum, G., Behnke, T., et al. 2007, Planet. Space Sci., 55, 928

Kaplan, G. H., Hughes, J. A., Seidelmann, P. K., Smith, C. A., \& Yallop, B. D. 1989, AJ, 97, 1197

Kolyuka, Y., Tikhonov, V., Ivanov, N., et al. 1991, A\&A, 244, 236

Lainey, V., Dehant, V., \& Pätzold, M. 2007, A\&A, 465, 1075

Lemmon, M., Bell, J., Malin, M., et al. 2013, EGU General Assembly Conf. Abstr., 15, 3732

Michael, G., \& Neukum, G. 2009, Lunar and Planetary Science Conference, 40, 1851
Morley, T. A. 1989, A\&AS, 77, 209

Oberst, J., Matz, K. D., Roatsch, T., et al. 2006, A\&A, 447, 1145

Oberst, J., Schwarz, G., Behnke, T., et al. 2008, Planet. Space Sci., 56, 473

Pasewaldt, A., Oberst, J., Willner, K., et al. 2012, A\&A, 545, A144

Pischel, R., \& Zegers, T. 2009, in Mars Express: the Scientific Investigations, ed. A. Chicarro, ESA SP, 1291, 249

Rambaux, N., Castillo-Rogez, J. C., Le Maistre, S., \& Rosenblatt, P. 2012, A\&A, 548, A14

Rosenblatt, P., Lainey, V., Le Maistre, S., et al. 2008, Planet. Space Sci., 56, 1043

Wählisch, M., Willner, K., Oberst, J., et al. 2010, Earth Planet. Sci. Lett., 294, 547

Wählisch, M., Stooke, P. J., Karachevtseva, I. P., et al. 2014, Planet. Space Sci., 102,60

Willner, K., Oberst, J., Wählisch, M., et al. 2008, A\&A, 488, 361

Willner, K., Oberst, J., Hussmann, H., et al. 2010, Earth Planet. Sci. Lett., 294, 541

Willner, K., Shi, X., \& Oberst, J. 2014, Planet. Space Sci., 102, 51

Witasse, O., Duxbury, T., Chicarro, A., et al. 2014, Planet. Space Sci., 102, 18

Zurek, R. W., \& Smrekar, S. E. 2007, J. Geophys. Res. Planets, 112, 5 